\title{
TH2C-1
}

\section{Data-Dependent Jitter and Crosstalk-Induced Bounded Uncorrelated Jitter in Copper Interconnects}

\author{
James Buckwalter, Behnam Analui, and Ali Hajimiri \\ California Institute of Technology, Pasadena, CA 91125
}

\begin{abstract}
This paper resolves the jitter impairment of non-return-to-zero data in transmission lines. The limited bandwidth of the transmission line introduces datadependent jitter. Crosstalk between neighboring lines results in bounded uncorrelated jitter in the data eye. An analytical approach to representing data-dependent jitter and crosstalkinduced bounded uncorrelated jitter is presented. Comparison with jitter measurements of microstrip lines on FR4 board demonstrates accuracy to within $15 \%$ of the predictions for deterministic jitter.
\end{abstract}

Index Terms - Jitter, Data-dependent Jitter, Bounded Uncorrelated Jitter, Interconnect, Crosstalk

\section{INTRODUCTION}

Exceptional effort is focused on extending the data rate capabilities of economical copper backplanes. While VLSI circuit speed is steadily increasing, the wires between chips and even on-chip are becoming bottlenecks [1][2]. To avoid costly optical interconnects, circuit designers are forced to equalize the channel to increase the data rate, implement alternative channel coding schemes, or simply use more parallel channels [3][4]. Ultimately, space and power dissipation limits the number of parallel channels.

Increasing the interconnect density in controlled impedance environments is a problem common to copper backplane and VLSI. Electromagnetic coupling between transmission lines is overwhelming as trace separation reduces. To prevent an aggressive neighboring signal from generating errors in a victim signal, circuit designers match line lengths such that the strongest coupling from the aggressor occurs not in the center of the data eye but during data transitions. While this prevents errors at the sampling point, the aggressor generates jitter on the victim line.

This paper studies the jitter penalty associated with coupling between neighboring microstrip lines. Jitter consists of deterministic and random components. In this paper, the important deterministic components are data-dependent jitter and bounded-uncorrelated jitter. First, the data-dependent jitter (DDJ) due to the transmission line is analyzed and a DDJ probability density function (PDF) is introduced. Fig. 1 illustrates the data transition ambiguity

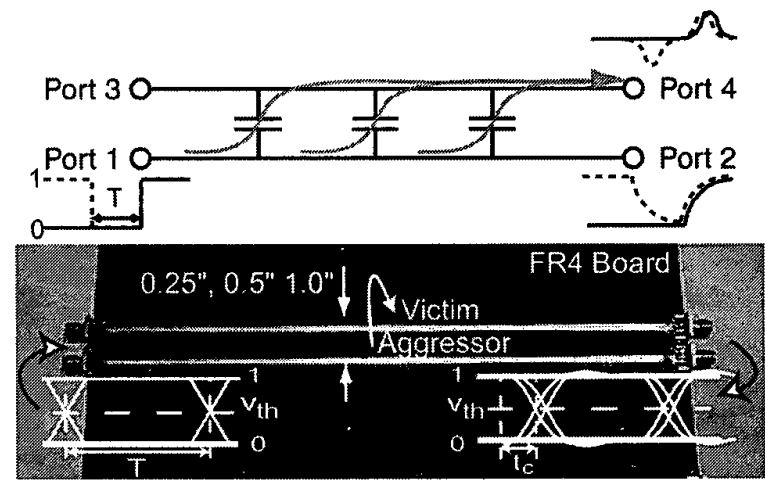

Fig. 1. The data-dependent jitter of a interconnect on FR4 board with various line separations. The illustration shows the threshold crossing time deviation through the transmission line and the capacitive coupling between neighboring lines.

caused by the microstrip line. Second, an aggressive neighboring signal causes bounded uncorrelated jitter (BUJ) and a PDF is formulated that accounts for the coupling. These two jitter PDFs dominate jitter in interconnects. The PDFs are predicted from the step response and S-parameters and matched to measurements on an FR4 board with microstrip lines of various separation.

\section{DATA-DEPENDENT JITTER IN COPPER INTERCONNECTS}

The properties of DDJ are determined analytically from a characterization of the transmission line. The received non-return-to-zero (NRZ) data signal is

$$
r(t)=\sum_{n=-\infty}^{\infty} a_{n} p(t-n T)
$$

where $a_{n}$ is the binary value and $p(t)$ is the pulse response with period $T$. The pulse response is related to the step response, $s(t)$;

$$
p(t)=s(t)-s(t-T) .
$$

Substituting (2) into (1), we have 


$$
r(t)=\sum_{n=-\infty}^{\infty} a_{n}[s(t-n T)-s(t-(n+1) T)]
$$

A first-order Taylor series approximates the step response.

$$
s(t-n T)=s\left(t_{o}-n T\right)+\left(t-t_{o}\right) s^{(1)}\left(t_{o}-n T\right)
$$

The superscript denotes the derivative order and $t_{o}$ is the time at which $s(t)$ crosses a voltage threshold, $v_{t h}$. Substituting (4) into (3), the threshold crossing time, $t_{c}$, is ${ }^{1}$

$$
t_{c}=t_{o}+\frac{v_{t h}-\sum a_{n}\left[s\left(t_{o}-n T\right)-s\left(t_{o}-(n+1) T\right)\right]}{\sum a_{n}\left[s^{(1)}\left(t_{o}-n T\right)-s^{(1)}\left(t_{o}-(n+1) T\right)\right]}
$$

Jitter is the deviation of the signal from a reference such as $t_{o}$ (i.e., $\Delta t_{c}=t_{c}-t_{o}$ ). Clearly, $t_{c}$ depends on earlier bits. Therefore, $\Delta t_{c}$ is the DDJ. If two previous bits are considered, there are four possible sequences with transitions at the current bit: $001,101,110$, and 010 . If we assume $v_{t h}=0.5$, there are two different $t_{c}$ for the 001 and 101 sequences as illustrated in Fig. 1. For 001 and 110 sequences, $t_{c}=t_{o}$ since, by definition, $s\left(t_{o}\right)=v_{t h}$. For the 101 and 010 sequences,

$$
\Delta t_{c, D D J}=\frac{1-s\left(t_{o}+T\right)}{s^{(1)}\left(t_{o}+T\right)-s^{(1)}\left(t_{o}\right)} .
$$

Notably, the denominator contains the slope implying that slow waveforms suffer from more DDJ. The firstorder DDJ is a two-valued PDF of $\Delta t_{c}$ as modelled in [5],

$$
p d f_{D D J}\left(\Delta t_{c}\right)=\frac{1}{2}\left[\delta\left(\Delta t_{c}\right)+\delta\left(\Delta t_{c}-\Delta t_{c, D D J}\right)\right] .
$$

If additional bits are considered, the PDF extends to more peaks. Both victim and aggressor signals suffer from the DDJ impairment because the transmission lines are identical.

\section{BOUNDED UNCORRELATED JITTER}

Capacitive coupling introduces energy from an aggressor to a victim signal as illustrated in Fig. 1. Inductive effects can also be considerable in certain cases. We assume capacitive coupling but inductive coupling can be treated similarly. The nature of the coupling capacitance, $C_{c}$, between microstrip lines has been studied (e.g. [6]).

1. Causality restricts the summation from $-\infty$ to 0 . These limits are assumed in the following summations.
Even and odd modes experience $C_{c}$ with relative strength of zero or two. For equiprobable NRZ data, the excitations are uncorrelated and both modes occur equally. Hence, the $C_{c}$ has a relative strength of one. Notably, the modedependent capacitance ultimately disperses the signal on the microstrip line.

Although the capacitance is distributed, the coupled signal travels through the same amount of transmission line irrespective of the coupling point, as shown in Fig. 1. Therefore, the coupling between the victim and aggressor line is approximated as a lumped capacitance:

$$
\left|S_{41}(\omega)\right| \approx \omega C_{c} Z_{o} / 2 .
$$

A differentiated version of the aggressor contributes to (3),

$$
r_{\text {coupled }}(t)=\frac{Z_{o} C_{c}}{2} \sum\left(b_{n}-b_{n-1}\right) s^{(1)}(t-n T) .
$$

The timing jitter resulting from the uncorrelated data sequence can now be calculated as

$$
\Delta t_{c, B U J}=-\frac{Z_{o} C_{c}}{2} \frac{\sum\left(b_{n}-b_{n-1}\right) s^{(1)}\left(t_{o}-n T\right)}{\sum\left(a_{n}-a_{n-1}\right) s^{(1)}\left(t_{o}-n T\right)},
$$

where $b_{n}$ is the binary value of the aggressor signal. Equation (10) features only the slope of the step response. Since a data transition occurs in the victim signal, $a_{0}-a_{-1}$ is always non-zero. Under certain cases, (10) simplifies further. If the aggressor and victim are identical, $t_{c, B U J}=-Z_{o} C_{c} / 2$. However, $C_{c}=0$ because the mode is even. If the aggressor is a differential signal, $a_{n}=-b_{n}$ and $t_{c, B U J}=Z_{o} C_{c}$ since the mode is odd. Therefore, the coupling delays the signal. For two uncorrelated bits, the role of the slope cancels and there are three values for $(10)$. Now, the PDF for the BUJ can be approximated.

$p d f_{B U J}=\frac{1}{4} \delta\left(\Delta t_{c}+\frac{Z_{o} C_{c}}{2}\right)+\frac{1}{2} \delta\left(\Delta t_{c}\right)+\frac{1}{4} \delta\left(\Delta t_{c}-\frac{Z_{o} C_{c}}{2}\right)(11)$

Convolving the PDF of the DDJ and BUJ determines the total deterministic jitter impairment of the received signal;

$$
p d f_{\text {Jitter }}\left(\Delta t_{c}\right)=p d f_{D D J}\left(\Delta t_{c}\right) \otimes p d f_{B U J}\left(\Delta t_{c}\right) .
$$

Since the first-order DDJ has two terms and the BUJ has three terms, the total deterministic jitter is a PDF with at most six delta functions corresponding to jitter peaks.

\section{EXPERIMENTAL RESULTS}

The FR4 board shown in Fig. 1 has 9" coupled microstrip lines separated by 0.25 ", 0.5 ", and 1 ". Network analy- 
sis of the lines is plotted in Fig. 2. $\mathrm{S}_{21}$ indicates that the $3 \mathrm{~dB}$ bandwidth is $2.5 \mathrm{GHz}$ for the 0.25 " lines and $3.8 \mathrm{GHz}$ for the 0.5 " and 1 " lines.

The measured step responses are plotted in Fig. 3 and are used to calculate the DDJ in Table I. The step response value is normalized to one in the table. The 0.25 " lines feature a frequency null near $9 \mathrm{GHz}$ and the step response is slowest. The negative value for the DDJ indicates that the 101 sequence is faster than the 001 sequence. Interestingly, while the 0.25 " line response in Fig. 3 is slowest, the DDJ calculated in Table $I$ is smallest. This emphasizes the importance of the slope after one period. Fig. 4,5, and 6 show the data eyes at $5 \mathrm{~Gb} / \mathrm{s}$ for the 0.25 ", 0.5 ", and 1.0 " lines without the impact of the aggressor signal. The DDJ values measured from these eyes are $6 \mathrm{ps}, 10 \mathrm{ps}$, and $10 \mathrm{ps}$. The DDJ measured from the data eyes agrees with the predicted DDJ value in (6).

\section{TABLE I}

Calculated and Measured DDJ for FR4 Coupled Microstrip

\begin{tabular}{|l|l|l|l|l|l|l|}
\hline Line & $s(t+T)$ & $s^{(1)}(t)$ & $s^{(1)}(t+T)$ & $\begin{array}{l}\text { Predicted } \\
\Delta t_{c, D D J}\end{array}$ & $\begin{array}{l}\text { Measured } \\
\Delta t_{c, D D J}\end{array}$ & Error \\
\hline $0.25 "$ & $0.98 \mathrm{~V}$ & $3.4 \mathrm{e} 9 \mathrm{~V} / \mathrm{s}$ & $-9.8 \mathrm{e} 7 \mathrm{~V} / \mathrm{s}$ & $-5.1 \mathrm{ps}$ & $-6 \mathrm{ps}$ & $15 \%$ \\
\hline $0.5 "$ & $0.95 \mathrm{~V}$ & $4.2 \mathrm{e} 9 \mathrm{~V} / \mathrm{s}$ & $5.7 \mathrm{e} 8 \mathrm{~V} / \mathrm{s}$ & $-11 \mathrm{ps}$ & $-10 \mathrm{ps}$ & $10 \%$ \\
\hline $1 "$ & $0.96 \mathrm{~V}$ & $4.5 \mathrm{e} 9 \mathrm{~V} / \mathrm{s}$ & $2.7 \mathrm{e} 8 \mathrm{~V} / \mathrm{s}$ & $-8.8 \mathrm{ps}$ & $-10 \mathrm{ps}$ & $12 \%$ \\
\hline
\end{tabular}

In Fig. 2, $\mathrm{S}_{41}$ shows the coupling is capacitive to $6 \mathrm{GHz}$. From ADS simulations, the coupling capacitance is $1.4 \mathrm{pF}$ for the 0.25 " line, $400 \mathrm{fF}$ for the 0.5 " line, and $120 \mathrm{fF}$ for the 1.0" line. The time constants that determine the PDF of the BUJ are therefore $35 \mathrm{ps}, 10 \mathrm{ps}$, and $3 \mathrm{ps}$ for these line separations, respectively, since the characteristic line impedance

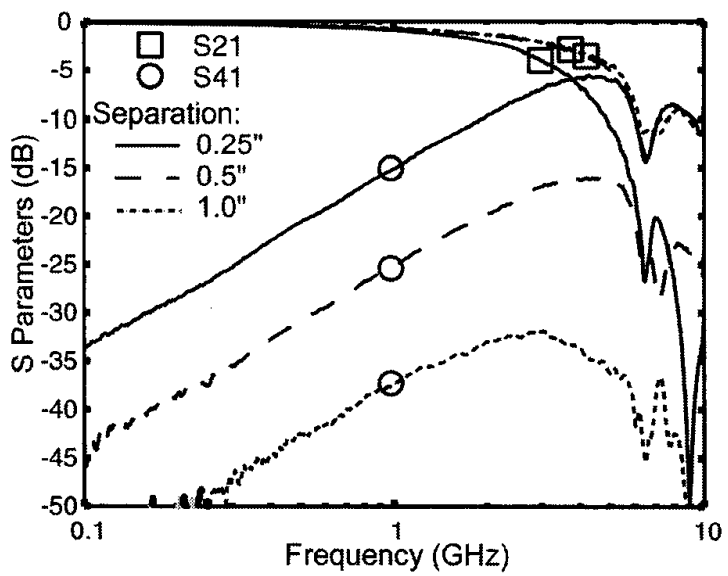

Fig. 2. Measurement of $S_{21}$ and $S_{41}$ for coupled microstrip line with varying microstrip separations.

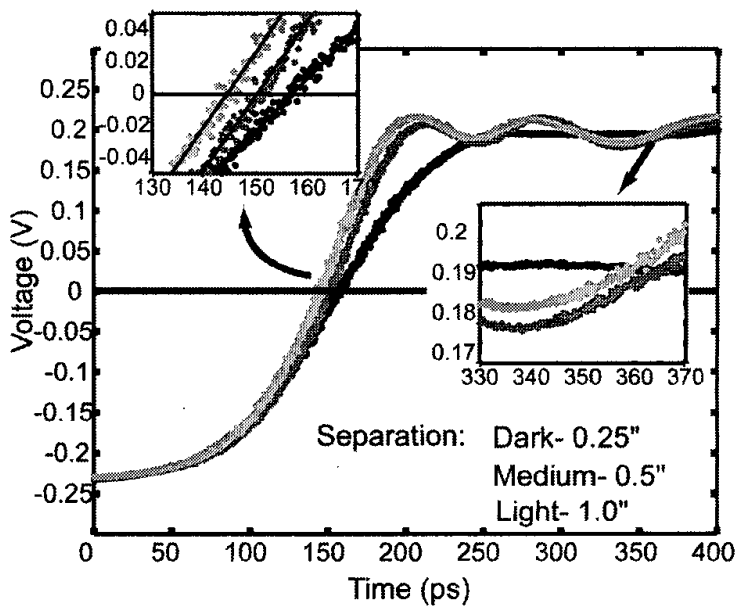

Fig. 3. Step response for different line separations. Inset graphs show slope at $t_{c}$ and at $t_{c}+T$.

is $50 \Omega$ The accuracy of the line impedance can have a drastic impact on the accuracy of the BUJ prediction.

Finally, the predicted values for the DDJ and the BUJ determine the location and probability of the jitter peaks given in (7) and (11) in the presence of an aggressive signal. A 5GB/s PRBS is introduced to the victim line. The differential line is delayed by four periods and introduced to the aggressor line. For the 0.25 " separation, the total deterministic jitter PDF is calculated in Table II. In this case, BUJ $>$ DDJ. Fig. 4 exhibits a central $\Delta t_{c}$ consisting of the $-5.1 \mathrm{ps}$ and $0 \mathrm{ps}$ terms. The random jitter limits the

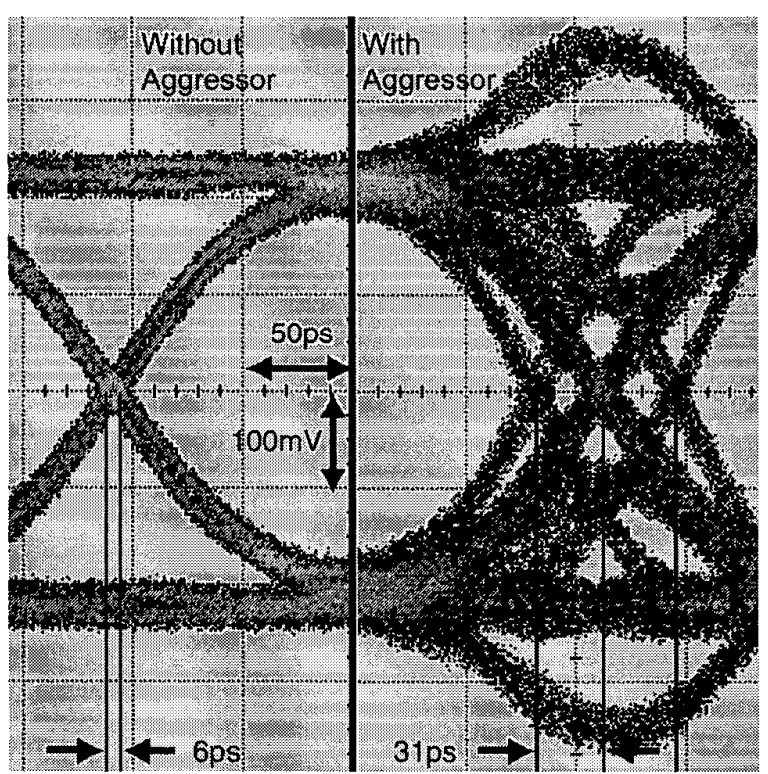

Fig. 4. Victim data eye with and without the aggressor signal at $5 \mathrm{~Gb} / \mathrm{s}$ for 0.25 " line separation. 


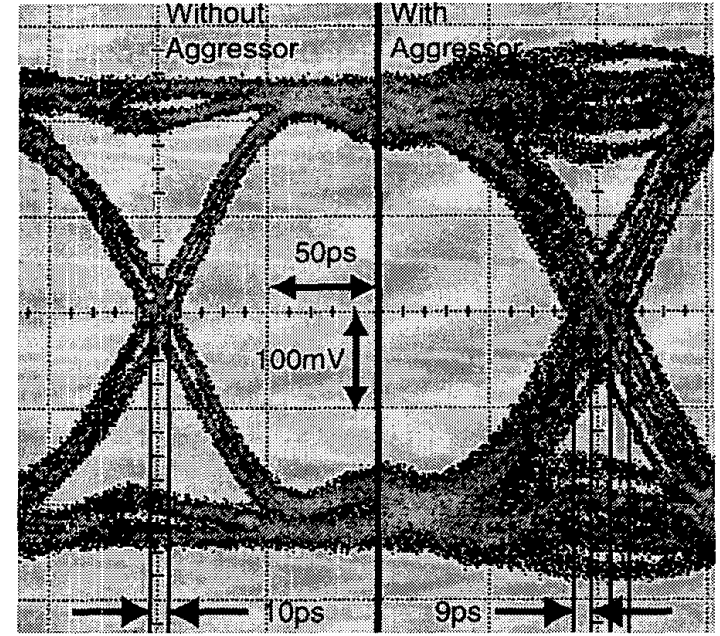

Fig. 5. Victim data eye for 0.5 " line separation.

resolution of the peaks. The probability indicates that the central threshold crossing occurs twice as often as the outer threshold crossings. The increased contrast of the central threshold crossing time in Fig. 4 confirms this. The peaks are separated by $31 \mathrm{ps}$ indicating that the overall error for the deterministic jitter prediction is about $11 \%$.

For the 0.5 " separation, the BUJ is comparable to the DDJ. More $\Delta t_{c}$ are obvious but the total range of the jitter is smaller. Again, random jitter prevents resolving the $11 \mathrm{ps}$ and -10 ps crossings and the $-1 \mathrm{ps}$ and $0 \mathrm{ps}$ crossings. Clearly, these peaks are more probable than the outer peaks in the Fig. 4 because they occur three times as often. The $\Delta t_{c}$ are separated by $9 \mathrm{ps}$. The predicted BUJ is $10 \mathrm{ps}$ so the error is about $10 \%$.

Finally, the 1" separation illustrates the case that the BUJ $<$ DDJ. The BUJ obscures the distinct $\Delta t_{c}$ of the DDJ and behaves like random jitter. As a result, verifying the predicted $\Delta t_{c}$ for the total deterministic jitter is difficult.

TABLE II

Predicted Location and Probability of Deterministic Jitter Peaks

\begin{tabular}{|c|c|c|c|}
\hline Line & $\Delta t_{c, D D J}$ & $\Delta t_{\boldsymbol{c}, \boldsymbol{B} U J}$ & $\Delta t_{c, D D J} \otimes B U J$ \\
\hline $0.25^{\prime \prime}$ & $-5.1 \mathrm{ps}$ & $35 \mathrm{ps}$ & $\begin{array}{l}-40 \mathrm{ps}(\operatorname{Pr}=1 / 8),-35 \mathrm{ps}(\operatorname{Pr}=1 / 8) \\
-5.1 \mathrm{ps}(\operatorname{Pr}=1 / 4), 0 \mathrm{ps}(\operatorname{Pr}=1 / 4), \\
\text { 30ps }(\operatorname{Pr}=1 / 8), 35 \mathrm{ps}(\operatorname{Pr}=1 / 8)\end{array}$ \\
\hline $0.5 "$ & $-11 \mathrm{ps}$ & 10ps & $\begin{array}{l}-21 \mathrm{ps}(\mathrm{Pr}=1 / 8),-11 \mathrm{ps}(\operatorname{Pr}=1 / 4), \\
-1 \mathrm{ps}(\operatorname{Pr}=1 / 8),-10 \mathrm{ps}(\operatorname{Pr}=1 / 8), \\
\text { Ops }(\operatorname{Pr}=1 / 4), 10 \mathrm{ps}(\operatorname{Pr}=1 / 8)\end{array}$ \\
\hline $1.0 "$ & $-8.7 \mathrm{ps}$ & $3 p s$ & $\begin{array}{l}-12 \mathrm{ps}(\operatorname{Pr}=1 / 8),-8.7 \mathrm{ps}(\operatorname{Pr}=1 / 4), \\
-5.7 \mathrm{ps}(\operatorname{Pr}=1 / 8),-3 \mathrm{ps}(\operatorname{Pr}=1 / 8), \\
0 \mathrm{ps}(\mathrm{Pr}=1 / 4), 3 \mathrm{ps}(\mathrm{Pr}=1 / 8)\end{array}$ \\
\hline
\end{tabular}

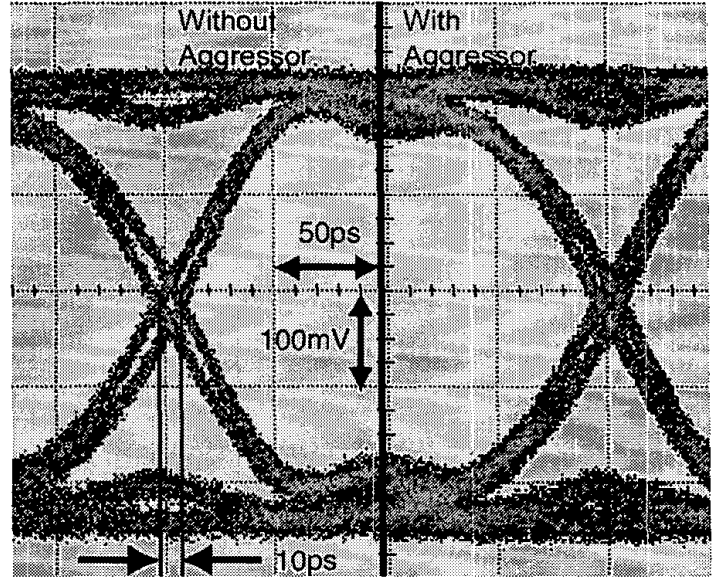

Fig. 6. Victim data eye for 1.0 " line separation

\section{CONCLUSIONS}

This paper develops an analysis of DDJ and BUJ in a coupled microstrip environment. The analysis of the DIJ emphasized the role of the step response slope in determining the DDJ impairment. The BUJ analysis stressed the coupling capacitance in determining the BUJ impairment. Predicted values for the data-dependent and bound uncorrelated jitter were calculated from the step response and Sparameter measurement of microstrip traces on an FR4 board. The observed data eyes agree to within $15 \%$ of the predicted DDJ and to $11 \%$ of the predicted BUJ. This gives a reasonable design rule for managing jitter degradation resulting from capacitive coupling.

\section{REFERENCES}

[1] R. Kollipara, G-J. Yeh, B. Chia, and A. Agarwal, "Design, Modeling and Characterization of High Speed Backplane Interconnects," DesignCon2003, Jan. 2003.

[2] R. Ho, K.W. Mai, and M.A. Horowitz, "The Future of Wires," Proc. of the IEEE, vol. 89, no. 4, pp.490-504, Apr. 2001.

[3] J. Zerbe, C. Werner, V. Stojanovic, F. Chen, J. Wei, G. Tsang, D. Kim, W. Stonecypher, A. Ho, T. Thrush, R. Kollipara, GJ. Yeh, M. Horowitz, K. Donnelly, "Equalization and Clock Recovery for a 2.5 - 10Gbs 2-PAM/4-PAM Backplane Transceiver Cell," ISSCC Digest, pp. 80-81, Feb. 2003.

[4] V. Stojanovic and M. Horowitz, "Modeling and Analysis of High-Speed Links," CICC 2003, pp.589-594, Sept. 2003.

[5] NCITS. Fibre Channel - Methodologies for Jitter Specification - MJSQ, T11.2/Project 1316-DT / Rev. 10.0. National Committee for Information Technology Standardization, March 2003.

[6] D. M. Pozar, Microwave Engineering, New York: J. Wiley \& Sons, 1998. 